\title{
OXIDATIVE STRESS BIOMARKERS IN TYPE 2 DIABETES MELLITUS FOR ASSESSMENT OF CARDIOVASCULAR DISEASE RISK
}

\author{
ROY ROBSON, AVINASH R KUNDUR, INDU SINGH* \\ School of Medical Science, Menzies Health Institute Queensland, Griffith University, Gold
}

Coast, Queensland, 4215, Australia.

\section{*Address correspondence to the author:}

Indu Singh,

School of Medical Science, G05_2.33, Griffith University, Gold Coast, Southport, Queensland, 4215, Australia.

Phone: +61 (0) 755529821

Fax: +61 (0) 755528087

E-mail: i.singh@griffith.edu.au

\section{Highlights}

- Increased oxidative stress in type-2 diabetes is associated with increased CVD risk

- Thrombosis, inflammation, vascular homeostasis and cellular proliferation act as major CVD risk factors

- Reactive oxygen species can significantly elevate CVD risk by promoting these risk factors via multiple pathways

\begin{abstract}
:
Aims: Type-2 Diabetes Mellitus (T2DM) is the most prevalent and progressive metabolic conditions affecting approximately $6.4 \%$ of the global population. Individuals with T2DM have a significantly increased risk of developing chronic conditions such as cardiovascular disease (CVD) and its associated complications, therefore, it is of great importance to establish strategies for combatting T2DM and its associated chronic conditions. Current literature has identified several biomarkers that are known to play a key role in the pathogenesis of CVD. Many of these biomarkers affecting CVD are influenced by an increase in oxidative stress as seen in T2DM. The purpose of this review is to analyse and correlate the oxidative stressrelated biomarkers that have been identified in the literature to provide an updated summary of their significance in CVD risk factors.
\end{abstract}


Data synthesis: This review has analysed current research on T2DM, CVD and oxidative stress. Four key cardiovascular risk factors: thrombosis, inflammation, vascular homeostasis and cellular proliferation were searched to identify potential biomarkers for this review. These biomarkers stem from six major cellular pathways; NF- $\kappa B$, Protein kinase-C, macrophage activation, arachidonic acid mobilization, endothelial dysfunction and advanced glycation end products.

Conclusions: The pathways and biomarkers were analysed to show their role as contributing factors to CVD development and a summary is made regarding the assessment of cardiovascular risk in T2DM individuals.

Key-words: Cardiovascular disease; oxidative stress; type-2 diabetes mellitus; thrombosis.

\section{Introduction}

Obesity and a sedentary lifestyle as part of urbanisation along with ageing are considered as one of the primary causes for a constant rise in type-2 diabetes mellitus (T2DM) [1]. In 2014, it was estimated that the world prevalence of diabetes was $8.5 \%$ of the global population. It has been predicted that by 2030 the incidence of diabetes will rise significantly, with an estimated $69 \%$ increase in developing countries especially, making diabetes the $7^{\text {th }}$ leading cause of mortality [2]. A vast range of chronic conditions are known to be associated with T2DM and cardiovascular diseases (CVD) being one of the primary causes of mortality within individuals [3].

Type-2 diabetes mellitus is characterised by chronically elevated blood glucose levels; this may be caused by increased insulin resistance and glucose intolerance. Chronic hyperglycaemia in T2DM can lead to increased oxidative stress and over time, it can generate a vicious cycle of reactive oxygen species (ROS) generation, thus, leading to alterations in vascular endothelium [4]. Elevated oxidative stress in T2DM individuals has been shown to be one of the major risk factors for an increased risk of CVD [5]. Increased free radical production has been shown to alter and induce several risk factors for CVD such as lipid peroxidation, endothelial dysfunction, inflammation and platelet activation [6]. Hence identifying the mechanisms and possible treatments to reduce the incidence of CVD has become a field of great significance. Chronic hyperglycaemia due to factors such as impaired glucose tolerance and insulin resistance can often lead to a range of chronic diseases in later life of individuals with T2DM [4]. 
As per a previous report, CVD can account for up to $65 \%$ of the mortality in individuals with T2DM. It is believed that the changes in an individual's thrombotic and inflammatory state through oxidative stress can be one of the causes for an increased mortality rates in T2DM [7]. These changes can individually or synergistically play a significant role in the elevating the risk of intravascular thrombosis and subsequent CVD [4]. Increased thrombogenicity, vascular cell proliferation, inflammation and reduced vascular homeostasis, may not only accelerate the progression of CVD but further contribute to the production of ROS in a positive feedback loop $[8]$.

\section{Oxidative stress and diabetes}

The current understanding of T2DM associated oxidative stress and its influence on vascular disease revolve around four key aspects and they are: thrombogenicity, vascular homeostasis, inflammation and cellular proliferation, as shown in Figure 1. Oxidative stress is an imbalance between the oxygen/nitrogen free radical production and the endogenous physiological antioxidant mechanisms [8]. Based on the literature, it is clear that the pathogenesis of CVD is multifactorial and therefore it is crucial identify specific ROS molecules and their associated pathways of production, in order to develop an effective and targeted therapy [9]. Glutathione, glutathione peroxidase (GPx), super oxide dismutase (SOD), and heme-oxygenase are some of the crucial antioxidant enzymes that negate the deleterious effects of excess free radicals [6]. In T2DM high blood glucose and free fatty acids (FFA) are responsible for an increase in ROS production due to metabolic stress resulting in changes within the electron transport chain (ETC) [10]. Literature shows that excessive mitochondrial ROS formation namely superoxide $\left(\mathrm{O}_{2}^{-}\right)$and hydrogen peroxide $\left(\mathrm{H}_{2} \mathrm{O}_{2}\right)$, is primarily due to the abnormalities seen in the mitochondrial respiratory chain when present in hyperglycaemic environments [11]. It is believed that this takes place in complex I of the ETC, through the activation of the receptor for advanced glycation end-products (RAGE) and a reduction in oxidative phosphorylation enzymes [12]. Previous research has shown that polyol pathway flux exacerbates intercellular oxidative stress and ROS interaction within the cell due to an increase in intracellular glucose and the hyperglycaemic environment found in T2DM [13]. The changes from the polyol pathway flux include increased enzyme activity of Aldose reductase resulting in excessive fructose, inactive alcohols and $\mathrm{NADP}^{+}$. This changed metabolic environment often leads to a 
decrease in cellular NADPH and thus a reduction in NADPH dependent antioxidants like glutathione, thus possibly aggravating the intercellular oxidative stress [14]. The compromise of physiological antioxidant defences may be a cause of increased oxidative stress in T2DM individuals [15]. It is believed that hyperglycaemia, glucose toxicity and excessive production of FFA's are the three main sources of diabetes related $\mathrm{NADP}^{+}$development. Furthermore, previous studies have shown hyperglycaemia directly contributes to atherosclerotic tissue damage through changes in the gene expression of endothelial cells caused by the increased hexamine pathway activity [16]. The increased production of FFA's alters metabolic pathways (insulin receptor substrate-1/PI3-Kinase/akt) increasing platelet aggregation and the production of oxidative free radicals exacerbating oxidative stress, ROS production and increasing the risk of CVD [17].

\section{Major Pathways Leading to Cardiovascular Disease}

Many studies have identified that the altered metabolic state associated with T2DM is one of the major issues causing an increase in oxidative stress and the subsequent changes in vascular homeostasis [8]. Evidence for literature has exemplified that antioxidants play a promising role in the field of preventative therapies [18]. Chronic hyperglycaemia and insulin resistance in T2DM has been associated with specific change in platelet physiology leading to an increase in platelet activation and aggregation [13]. Increase in platelet activation can occur through multiple stimulating factors, where oxidative stress has been identified as one of the key contributor to positively influence these activation pathways [8]. Numerous studies have already shown the ability of free radicals $\left(\mathrm{O}_{2}^{-}, \mathrm{H}_{2} \mathrm{O}_{2}, \mathrm{NADPH}\right.$ Oxidase; NOX) to increase cellular adhesion molecules such as P-selectin, PCAM, E-selectin and VCAM-1 on platelet and endothelial cell surfaces. P-Selectin is a cellular adhesion molecule released from activated platelet and endothelial cells; it is known to play a key role in leukocyte recruitment and the formation of large thrombus [19, 20]. Similarly E-Selectin, an endothelial-leukocyte adhesion molecule, is overexpressed under elevated oxidative stress and inflammatory states. Other thrombogenic factors associated with an increase in oxidative stress include arachidonic acid mobilization, thromboxane A2 and prostaglandin (PG) production [21]. On an endothelial level, oxidative stress has shown to directly increase vessel wall damage and disruption to the endothelium [13]. Furthermore, oxidative stress also directly reduces endothelial nitric oxide synthase (eNOS) activity, thereby reducing Nitric oxide (NO) production[22]. Nitric oxide is a key platelet aggregation inhibitor and is known to play a significant role in maintaining vascular homeostasis [23]. High levels of $\mathrm{H}_{2} \mathrm{O}_{2}$ and $\mathrm{O}_{2}{ }^{-}$generated from and over active NOX, 
can perforate the lipid bilayer of endothelial cells releasing inflammatory and apoptotic cytokines that lead to a cascade of thrombogenic and inflammatory events [24]. Damaged endothelial cells also lead to the exposure of collagen where glycoprotein receptors on the platelet surface interact with the damaged endothelium, stimulating platelet activation and aggregation at the site, thus promoting thrombotic and inflammatory activity [13].

Atherosclerosis, is an inflammatory condition that is associated with plaque formation and narrowing of arteries. Increased vascular inflammation is considered as one of the primary steps in the initiation of atherosclerosis, as it eases the passage of macrophages into the vessel endothelium and triggers that formation of foam cells [25]. Many pro-inflammatory pathways including Nuclear factor kappa-light-chain-enhancer of activated B cells (NF- $\kappa \mathrm{B})$ and Mitogen-activated protein kinase (MAP-kinase) elevate $\mathrm{O}_{2}{ }^{-}$levels, leading to the subsequent increase in peroxynitrite $\left(\mathrm{ONOO}^{-}\right)$as seen in T2DM [26]. The activation of inflammatory pathways can occur via multiple mechanisms throughout the cell, however the synergistic effect of these radical oxidant activated pathways along with hyperglycaemic conditions in T2DM individuals can lead to chronic inflammatory state, which can be pathogenic in nature. Endothelial dysfunction/deregulation often converges with white cell migration and the already disturbed state of the vasculature, enhancing the problem of atherosclerosis [27]. Excessive oxidative stress also increases endothelial and vascular smooth muscle cell proliferation through the increase of NF- $\kappa \mathrm{B}$, Transforming growth factor alpha (TGF- $\alpha$ ) and Transforming growth factor beta (TGF- $\beta$ ) [5]. Excessive cellular proliferation significantly adds to the pathology of atherosclerosis by rapidly increasing atherogenesis. Figure 2 shows a detailed overview of the major cellular pathways affected by oxidative stress in CVD. The main pathways are stemming from macrophage activation, methylglyoxal/AGEs, cellular adhesion molecules/endothelial dysfunction, protein kinase-C, peroxynitrite/arachidonic acid and NF$\kappa \mathrm{B}$. As shown in Figure 2, the presence of oxidative stress alters the thrombotic, inflammatory, cellular proliferation and homeostatic pathways. The figure aims to show an overall understanding of the concepts discussed in this review, exemplifying the cellular oxidative stress pathways involved in the pathogenesis of CVD.

\section{Methylglyoxal and Advanced Glycation End Products}

Advanced glycation end products (AGE's) are formed from sugar derived substances, gradually accumulating over time. However, in T2DM, this process is rapidly accelerated by the hyperglycaemic environment in conjunction with elevated oxidative stress, leaving the 
individual more susceptible to atherosclerosis and CVD [28]. Methylglyoxal and AGE has been known to induce apoptosis/necrosis and thrombosis, implicating them as major contributing factors in CVD and atherosclerotic plaque formation [29]. Alterations in metabolites found in T2DM increase the production of AGE's like diacyglycerol, 3-deoxyglucoseone, glyceraldehyde's methylglyoxal and glycoaldehyde. These metabolites can be attributed to the changes found in the glyoxal and polyol pathways that have been altered due to the presence of oxidative/glycaemic environments [13]. An increase in AGE's furthermore triggers an increase in the expression of receptor of AGE (RAGE). The RAGE can be found on endothelial cells and vascular smooth muscle cells, it is understood that with activation of AGE-RAGE binding, downstream cellular effects can occur leading to an increase in the production of altered substrates. Hyperglycaemia is a key contributor to the elevation of AGE's as the altered metabolic state promotes the increase of glycated proteins and lipids [30].

Methylglyoxal, which is a by-product of several metabolic pathways has been identified to play a key role in the accumulation of AGE's and elevating cellular oxidative stress, thereby contributing in the development of arterial atherogenesis [13]. The result of AGE-RAGE binding triggers the activation of ROS sensitive biochemical pathways and increases in TGF$\alpha$ and TGF- $\beta$, promoting vascular cell proliferation [31]. Furthermore, the activation of the AGE-RAGE pathway also activates the transcription factor NF- $\kappa B$, thus increasing $\mathrm{O}_{2}{ }^{-}$and inflammatory cytokine production. The activation of ROS sensitive biochemical pathways disrupts the regulation of vascular homeostatic enzymes such as eNOS and increase intracellular O-Linked $\beta$-N-acetylglucosamine (O-GlcNAc) [32]. O-GlcNAc is an intracellular single sugar modification on the hydroxyl moieties of amino acids serine and threonine. An increase in O-GlcNAc is associated with an elevated risk of intravascular thrombosis by reducing NO bioavailability via inhibiting eNOS function, upregulating plasminogen activator inhibitor-1 (PAI-1). Plasminogen activator inhibitor-1 (PAI-1) is an inhibitor of tissue plasminogen activator (tPA), which is central to the breakdown of blood clots, elevated levels of PAI-1 is associated with an increased risk of thrombosis [33]. Homeostasis is also affected by the deregulation of other vascular homeostatic enzymes and proteins (NF- $\kappa B$ ) [5]. Elevation of AGE's and the presence of hyperglycaemia not only effects homeostatic and thrombotic functions in the vessel, but can reduce the enzymatic antioxidant capacity used in the cellular defence mechanisms [31].

\section{Macrophage activation}


Oxidation of low density lipoprotein (LDL) by free radicals, also known as lipid peroxidation, is considered one of the hallmark risk factors for CVD. Several studies have shown a direct relationship between T2DM, oxidative stress and Oxidised LDL (OxLDL) [34]. Studies have shown that the excessive oxLDL within the vessel endothelium leads to the expression of monocyte chemo attractant protein-1 (MCP-1). MCP-1 is a chemoattractant known to initiate macrophage migration and foam cell formation [35]. The atherosclerotic foam cells further promote disruptions to the vessel endothelium and increasing thrombogenicity by enabling mobilisation of arachidonic acid (AA) [36]. The influx of activated macrophages and foam cells leads to a cascade of inflammatory and thrombotic pathways exacerbated in elevated oxidative stress environments [37]. Activated macrophages along with elevated oxidative stress further promote cyclooxygenase-1 (COX-1) enzymatic activity and inflammatory cytokines [38]. An increase in COX-1 coincides with an increase in AA, as COX-1 can metabolise AA into $\mathrm{PG} \mathrm{G}_{1} / \mathrm{H}_{1}$ to be further converted to thromboxane $\mathrm{A} 2$, prostacyclin $\mathrm{I}_{2}$ and $P G E_{2}, D_{2}, F_{2}$ causing altered platelet aggregation and activation [39]. Macrophage activation has also been shown to increase free AA production within the foam cell thus further promoting the thrombotic and deregulatory pathways seen in atherosclerosis [38]. Macrophage activation is also associated with an increase in the production of inflammatory cytokines such as Interleukin 1 Beta (IL-1 $\beta$ ), Interleukin 6 (IL-6), Interleukin 8 (IL-8), Interleukin 23 (IL-23), Tumour necrosis factor Alpha (TNF- $\alpha$ ) and Interferon I (INF-I) [40]. Excessive inflammation is marked by $\mathrm{C}$ reactive protein (CRP), an acute phase protein produced in hepatic cells, enabling up-regulation of IL-6 through autocrine and paracrine signalling to macrophages [41]. An increased endothelial CRP was shown to be associated with increased platelet activity as well as upregulating cellular and platelet adhesion. This further enables pro-inflammatory processes increasing the production of fibrinogen from monocytes within the vasculature enhancing the thrombogenic state [42]. The upregulation of CRP also increases tissue factor production, activating the extrinsic blood coagulation pathway. Increased adhesion of macrophages upregulates transcription factor NF- $\mathrm{KB}$, and it has recently been identified that NF- $\kappa \mathrm{B}$ plays a substantial role in the enhancement of platelet aggregation [43]. The upregulation of activated NF- $\mathrm{KB}$ furthermore leads to increased smooth muscle and vascular cell inflammation through the increase of cytokines like IL-6 [13]. The initial activation of macrophages increases the cellular reactive nitrogen species $\left(\mathrm{ONOO}^{-}\right)$and adds to the intracellular oxidative stress [38].

\section{NF- $\kappa B$}


The nuclear factor kappa-light-chain-enhancer of activated B cells is a key cellular response protein, involved in cytokine production and a regulator of specific DNA transcription [43]. $\mathrm{NF}-\kappa \mathrm{B}$ primarily serves as a regulator of cellular control in response to cellular stress factors, i.e. oxidative stress, inflammatory cytokines [44]. The NF- $\kappa B$ pathway is believed to be the key driving force behind inflammatory pathways within the vascular endothelium in the presence of oxidative stress [45]. Inflammation in conjugation with a state of elevated oxidative stress can accelerate the pathogenesis of vascular disease largely due to its role in foam cell formation in atherosclerosis. Under excessive oxidative stress conditions it is understood that the p65 subunit of NF- $\mathrm{KB}$ is activated and upregulated leading to both increased cytokine production and gene encryption of monocyte chemoprotein-1 (MCP-1)/vascular cell adhesion molecule-1 (VCAM-1) [44]. The increase in MCP-1/VCAM-1 is directly associated with an increase in TNF- $\alpha$ and monocyte adhesion to the endothelium, both factors promoting inflammation. The cytokines and growth factors implicated, include angiotensin II, IL-1 $\beta$, endothelial growth factor (EGF), platelet derived growth factor (PDGF), transforming growth factor (TGF), macrophage-activating factor-1 and TNF- $\alpha$ [46].

\section{Keap1-Nrf2}

In addition to NF- $\kappa \mathrm{B}$, the Keap1-Nrf2 pathway is also a critical transcriptional pathway. The Kelch-like ECH-associated protein 1 (Keap1)-Nuclear factor (erythroid-derived 2)-like 2 (Nrf2) pathway plays a crucial role in the regulation of the environmental stress response. In the Keap1-Nrf2 system, Keap1 is responsible for the ubiquination of Nrf2 under normal conditions. However, when oxidative stress (ie. $\mathrm{OONO}^{-}$and the resulting $\mathrm{O}_{2}^{-}$in $\mathrm{T} 2 \mathrm{DM}$ ) is present, Keap1 loses its ability to ubiquinate the nuclear factor, leading to its activation [47]. Once Nrf2 is activated it responds by enhancing gene expression of a vast number of genes, including those that regulate antioxidant and detoxification enzymes as well as inflammatory responses [32]. Therefore, an increase in the Keap1-Nrf2 pathway would indicate increased levels of intracellular oxidative stress as seen in T2DM. In individuals with T2DM it has been identified that enhanced levels of Nrf2 are present in the kidneys suggesting that increased Nrf2 levels is a physiological adaptive response when oxidative stress is at play [48]. Studies have shown that the activation of the Keap1-Nrf2 pathway specifically reduces inflammatory regulators such as NF- $\mathrm{BB}$ and thrombotic activators such as VCAM-1 [48]. The activation of Nrf2 aids in achieving an oxidative stress balance. A recent animal study by Bhakkiyalakshmi et al. showed that elevated levels of activated Nrf2 in a murine model lead to a reduction in lipid peroxidation and disturbances in the lipid profile [49]. It has also been shown that in the 
absence of $\mathrm{Nrf} 2$, the risk of vascular complications in diabetes is increased [48]. Studies have suggested that the enhancement of the Keap1-Nrf2 system, may be useful as pharmacological intervention [32]. Furthermore, current research has shown that activation of the Keap1-Nrf2 pathway may be particularly helpful in combatting the deleterious effects of hyperglycaemic stress seen in disorders like T2DM [50].

\section{Cellular adhesion molecules and endothelial dysfunction}

Many cellular adhesion molecules play significant roles in the pathogenesis of CVD. With correct identification they have the potential to be used as cellular biomarkers for an increased pro-thrombotic susceptibility. Cellular adhesion molecules such as intra-cellular adhesion molecule 1 (ICAM-1), VCAM-1, P-Selectin and E-Selectin moderate the interactions between thrombotic factors and the vessel endothelium [51]. It is believed that elevated oxidative stress contributes to an increase in the expression of these adhesion molecules. Furthermore, hyperglycaemia has also been implicated in this molecular activity due to the altered metabolites found in T2DM [52]. Endothelial expression of these adhesion molecules plays a major role in platelet aggregation and the atherogenesis. Studies have shown that with the increase in expression of P-Selectin and E-Selectin, elevated platelet-fibrin and plateletleukocyte aggregation ensues thrombogenic activity [19]. The elevated ICAM-1 and VCAM1 at the endothelial cell also contribute to the increased state of platelet aggregation and adhesion. Research has also shown that the increase of ICAM-1 and VCAM-1 allows for enhanced white cell migration into the vasculature through rolling adhesion to vascular cells in atherosclerosis formation [53]. Dysfunction of the endothelial cell lining reduces crucial enzymatic activity e.g. eNOS, reducing NO and further promoting platelet aggregation. The increased oxidative stress seen in T2DM has shown to increase surface glycoproteins such as ICAM-1 and VCAM-1 in the vasculature. In normal physiology these molecules mediate inflammation, intracellular signalling, and immune responses. However, in T2DM the increased levels of $\mathrm{H}^{2} \mathrm{O}^{2}$ and $\mathrm{O}^{2}$. lead to the elevated adhesion to both platelets and white cells to the endothelial monolayer through rolling adhesion. This increased adhesion leads to promotion of white cell migration in to the endothelium, foam cell formation and platelet aggregation [54]. These mechanisms increase the atherosclerotic and thrombotic tendencies seen in CVD within T2DM individuals.

\section{Protein Kinase-C}


A large and growing body of literature is implicating protein kinase-C (PK-C) as a major activator both thrombotic and homeostatic pathways $[55,56]$. The increased intracellular PK$\mathrm{C}$ concentration and its activation is believed to arise from multiple hyperglycaemic and oxidative stress related pathways. One of the major activating pathways of PK-C occurs though increased intracellular $\mathrm{H}_{2} \mathrm{O}_{2}$ present in T2DM. The increase in $\mathrm{H}_{2} \mathrm{O}_{2}$ leads to the increase of NOX and the subsequent increase in PK-C activity. The increased activity of PK-C is further promoted though the increase of diacylglycerol and/or a change in calcium ions induced though increased cellular oxidative stress, namely $\mathrm{O}_{2}^{-}[13]$. The activation of PK-C works as a proteinphosphorylating enzyme, the phosphorylation PK-C can alter the outcome of signal transduction pathways. It has been indicated that PK-C is a mediator of cellular function, inflammation and vasculature homeostasis. Activation of PK-C can be seen in vascular smooth muscle and platelets activating serotonin g-protein coupled receptor signalling, causing platelet aggregation and changes to the vascular homeostasis [57]. Under oxidative stress environments a direct upregulation of PK-C has previously been identified, as PK-C is a key cell mediator. This increase has significant effects in CVD progression [5]. Research investigating oxidative stress related up regulation of PK-C has identified direct changes to Mitogen-activating Protein-Kinase (MAP-K), eNOS, COX-2 and endothelin-1 (ET-1) [13]. Enhanced platelet activation can be seen through both reductions in eNOS and through the COX 2 pathway. Increases in PK-C have been correlated with a direct reduction of eNOS leading to a reduction in NO bioavailability [58].

PK-C contributes to increased thrombotic state through the up regulation of COX-2 in the endothelial cell, COX-2 enzymatic activity increases prostacyclin and thromboxane A2 [59]. The increase in prostacyclin alters vascular homeostasis and inhibition of platelet activation. Meanwhile thromboxane A2 production via the COX-2 pathway can increase platelet activation and aggregation despite the prostacyclin production [60]. Upregulation of COX-2 by PK-C has a direct correlation to an increase of vascular inflammation [59]. Additionally the increase in PK-C enhances the expression of the P38 subunit of MAP-K, further contributing to vascular inflammation through cytokine release, increasing ET-1 and vasoconstriction, promoting a loss in vascular homeostasis [61].

Recent studies have outlined PK-C as a potential therapeutic target for the treatment of cardiovascular disease in a human model [62]. To date, there have been numerous clinical studies have investigated the effects of PKC inhibitors/inhibition on murine models showing a reduced risk in CVD risk factors [63, 64] Furthermore, several clinical studies have linked PK- 
$\mathrm{C}$ inhibition to assist in the attenuation of the diabetes related neuropathy and nephropathy, with some studies showing promising results in CVD $[65,66]$. In addition, there are a number of studies that relate to the reduction of PK-C activity though inhibition of NADPH oxidase [67].

\section{Peroxynitrite and Arachidonic Acid}

Peroxynitrite $\left(\mathrm{ONOO}^{-}\right)$is a highly unstable reactive nitrogen species formed through the elevation of ROS primarily $\mathrm{O}_{2}^{-}$found in T2DM, the increased oxidative stress increases the susceptibility of NO oxidization by hyperglycaemia induced superoxide $\left(\mathrm{O}_{2}^{-}\right)$[23]. Peroxynitrite is a powerful oxidative molecule that can perforate the phospholipid bilayer and cause damage to other cellular structures either destroying the cell or disrupting cellular functions. Furthermore, research has shown that $\mathrm{ONOO}^{-}$has a key role in eNOS uncoupling [68], which leads to a reduced NO capacity and endothelial dysfunction [69]. Several studies have identified effect of oxidative stress on NO [70], and as NO is a key modulator of clotting in vivo, it is of great importance in the synopsis of CVD. To date, previous studies have shown that regulation of platelet aggregation occurs through cyclic guanosine monophosphate (cGMP) in NO regulation, providing a powerful anti-aggregation mechanism when in sufficient quantities [71]. However in diseases like T2DM, production of NO is reduced leading to platelet-endothelium adhesion, triggering platelet aggregation and thrombi formation [70]. Furthermore several studies have documented that reduced NO availability is directly linked with adverse cardiovascular outcomes [72]. Reduction's in NO furthermore contributes to atherogenic activity by increasing oxLDL, disruptions to vascular homeostasis monocyte adhesion and smooth muscle cell proliferation, making it a key element to the development of atherosclerosis [73]. Unstable metabolites, in combination with elevated quantities reactive species including $\mathrm{ONOO}^{-}, \mathrm{O}_{2}{ }^{-}$and hydrogen peroxide $\left(\mathrm{H}_{2} \mathrm{O}_{2}\right)$ are found in individuals with diabetes, this leads to further destruction of the phospholipid bilayer increasing free AA through the activation of Phospholipase $A_{2}$ [74]. Arachidonic acid is a fundamental part of the phospholipid membrane, when perforated or destroyed excessive amounts of AA is mobilised. When AA is mobilised conversion to Prostaglandin $\mathrm{G}_{2}\left(\mathrm{PGG}_{2}\right)$ occurs through $\mathrm{COX} 1$ and $\mathrm{COX} 2, \mathrm{PGG}_{2}$ is then converted to Prostaglandin $\mathrm{H}_{2}\left(\mathrm{PGH}_{2}\right)$. The rise in $\mathrm{PGH}_{2}$ then increases prostacyclin and thromboxane A2 [10]. The conversion of $\mathrm{PGH}_{2}$ functions as a key activator of platelets and disruption to the vascular homeostasis. Free AA is also metabolised by 5lipoxyginase to 5-hydroperoxyeicosatetraenoic acid (5-HTEPE), promoting a cascade of leukotrienes $\left(\mathrm{A}_{4}, \mathrm{C}_{4}, \mathrm{E}_{4}, \mathrm{D}_{4}\right)$ increasing vasodilation and vascular permeability [36]. 


\section{Conclusion}

As the incidence of $\mathrm{T} 2 \mathrm{DM}$ is ever on the rise, the prevalence of related pathologies are continually increasing. By summarising and identifying the biomarkers reviewed in this paper, a better diagnostic and therapeutic treatment can be established. This review gives an updated summary of the major pathways and significant markers associated with atherosclerosis and CVD in T2DM. Novel methods in the assessment and identification of people with diabetes atrisk of cardiovascular diseases like atherosclerosis and thromboembolisms can be identified. This review will aid health professionals and researchers in profiling individuals with diabetes in their relevant CVD risk categories, allowing for quicker and more effective treatment by targeting specific pathways. The ability to collaboratively identify the changes caused from oxidative damage in thrombotic factors, vascular inflammation, cellular proliferation and reduced vascular homeostasis will further help researchers in identifying pathways in the processes of CVD prevalence and the associated risk for T2DM individuals.

\section{Summary}

Figure 3 is a summary of the effects of oxidative stress on thrombosis, inflammation, vascular cell proliferation and vascular homeostasis. As shown, many factors can contribute to more than one category of the atherosclerotic development (PK-C) where as others are more specific to one pathway (ONOO-; thrombosis, NF- $\kappa \mathrm{B}$; inflammation, TGF-; vascular cell proliferation and O-GlcNAcylation; vascular homeostasis).

\section{References}

1. Hu FB. Globalization of Diabetes: The Role of Diet, Lifestyle, and Genes. Diabetes Care. 2011;34(6):1249-1257.

2. Organization WH. Global Report on Diabetes. World Health Organization; 2016.

3. Fukuoka Y, Choi J, M SB, Gonzalez P, Arai S. Family History and Body Mass Index Predict Perceived Risks of Diabetes and Heart Attack among Community-Dwelling Caucasian, Filipino, Korean, and Latino Americans-Dilh Survey. Diabetes Res Clin Pract. 2015;109(1):157163.

4. Salas-Salvado J, Martinez-Gonzalez MA, Bullo M, Ros E. The Role of Diet in the Prevention of Type 2 Diabetes. Nutr Metab Cardiovasc Dis. 2011;21 Suppl 2:B32-48.

5. Giacco F, Brownlee M. Oxidative Stress and Diabetic Complications. Circ Res. 2010;107(9):1058-1070.

6. Hopps E, Noto D, Caimi G, Averna MR. A Novel Component of the Metabolic Syndrome: The Oxidative Stress. Nutr Metab Cardiovasc Dis. 2010;20(1):72-77. 
7. American Diabetes A. Peripheral Arterial Disease in People with Diabetes. Diabetes Care. 2014;26(12):3333-3341.

8. Pitocco D, Tesauro M, Alessandro R, Ghirlanda G, Cardillo C. Oxidative Stress in Diabetes: Implications for Vascular and Other Complications. Int J Mol Sci. 2013;14(11):21525-21550.

9. Forman HJ, Ursini F, Maiorino M. An Overview of Mechanisms of Redox Signaling. J Mol Cell Cardiol. 2014;73:2-9.

10. Parente L. Pros and Cons of Selective Inhibition of Cyclooxygenase-2 Versus Dual Lipoxygenase/Cyclooxygenase Inhibition: Is Two Better Than One? J Rheumatol. 2001;28(11):2375-2382.

11. Rolo AP, Palmeira CM. Diabetes and Mitochondrial Function: Role of Hyperglycemia and Oxidative Stress. Toxicol Appl Pharmacol. 2006;212(2):167-178.

12. Coughlan MT, Thorburn DR, Penfold SA, Laskowski A, Harcourt BE, Sourris KC, et al. RageInduced Cytosolic Ros Promote Mitochondrial Superoxide Generation in Diabetes. J Am Soc Nephrol. 2009;20(4):742-752.

13. Paneni F, Beckman JA, Creager MA, Cosentino F. Diabetes and Vascular Disease: Pathophysiology, Clinical Consequences, and Medical Therapy: Part I. Eur Heart J. 2013;34(31):2436-2443.

14. Sies H, Berndt C, Jones DP. Oxidative Stress. Annu Rev Biochem. 2017.

15. Bhatia S, Shukla R, Venkata Madhu S, Kaur Gambhir J, Madhava Prabhu K. Antioxidant Status, Lipid Peroxidation and Nitric Oxide End Products in Patients of Type 2 Diabetes Mellitus with Nephropathy. Clin Biochem. 2003;36(7):557-562.

16. Stadler K. Oxidative Stress in Diabetes. Adv Exp Med Biol. 2012;771:272-287.

17. Gastaldelli A, Basta G. Ectopic Fat and Cardiovascular Disease: What Is the Link? Nutr Metab Cardiovasc Dis. 2010;20(7):481-490.

18. Riccioni G, Speranza L, Pesce M, Cusenza S, D'Orazio N, Glade MJ. Novel Phytonutrient Contributors to Antioxidant Protection against Cardiovascular Disease. Nutrition. 2012;28(6):605-610.

19. Santhakumar AB, Stanley R, Singh I. The Ex Vivo Antiplatelet Activation Potential of Fruit Phenolic Metabolite Hippuric Acid. Food Funct. 2015;6(8):2679-2683.

20. Freedman JE. Oxidative Stress and Platelets. Arterioscler Thromb Vasc Biol. 2008;28(3):s1116.

21. Balboa MA, Balsinde J. Oxidative Stress and Arachidonic Acid Mobilization. Biochim Biophys Acta. 2006;1761(4):385-391.

22. A. Cerielloa RT, S. Genovesed, . Clinical Implications of Oxidative Stress and Potential Role of Natural Antioxidants in Diabetic Vascular Complications. Nutrition, Metabolism and Cardiovascular Diseases. 2016.

23. Gkaliagkousi E, Corrigall V, Becker S, de Winter P, Shah A, Zamboulis C, et al. Decreased Platelet Nitric Oxide Contributes to Increased Circulating Monocyte-Platelet Aggregates in Hypertension. Eur Heart J. 2009;30(24):3048-3054.

24. Drummond GR, Selemidis S, Griendling KK, Sobey CG. Combating Oxidative Stress in Vascular Disease: Nadph Oxidases as Therapeutic Targets. Nat Rev Drug Discov. 2011;10(6):453-471.

25. Dall'Asta M, Derlindati E, Ardigo D, Zavaroni I, Brighenti F, Del Rio D. Macrophage Polarization: The Answer to the Diet/Inflammation Conundrum? Nutr Metab Cardiovasc Dis. 2012;22(5):387-392.

26. Folli F, Corradi D, Fanti P, Davalli A, Paez A, Giaccari A, et al. The Role of Oxidative Stress in the Pathogenesis of Type 2 Diabetes Mellitus Micro- and Macrovascular Complications: Avenues for a Mechanistic-Based Therapeutic Approach. Curr Diabetes Rev. 2011;7(5):313324.

27. Mudau M, Genis A, Lochner A, Strijdom H. Endothelial Dysfunction: The Early Predictor of Atherosclerosis. Cardiovasc J Afr. 2012;23(4):222-231. 
28. Kalousova M, Skrha J, Zima T. Advanced Glycation End-Products and Advanced Oxidation Protein Products in Patients with Diabetes Mellitus. Physiol Res. 2002;51(6):597-604.

29. Kim J, Kim OS, Kim CS, Sohn E, Jo K, Kim JS. Accumulation of Argpyrimidine, a MethylglyoxalDerived Advanced Glycation End Product, Increases Apoptosis of Lens Epithelial Cells Both in Vitro and in Vivo. Exp Mol Med. 2012;44(2):167-175.

30. Jandeleit-Dahm K, Watson A, Soro-Paavonen A. The Age/Rage Axis in Diabetes-Accelerated Atherosclerosis. Clin Exp Pharmacol Physiol. 2008;35(3):329-334.

31. Yamagishi S. Role of Advanced Glycation End Products (Ages) and Receptor for Ages (Rage) in Vascular Damage in Diabetes. Exp Gerontol. 2011;46(4):217-224.

32. Rochette L, Zeller M, Cottin Y, Vergely C. Diabetes, Oxidative Stress and Therapeutic Strategies. Biochim Biophys Acta. 2014;1840(9):2709-2729.

33. Groves JA, Lee A, Yildirir G, Zachara NE. Dynamic O-Glcnacylation and Its Roles in the Cellular Stress Response and Homeostasis. Cell Stress Chaperones. 2013;18(5):535-558.

34. Njajou OT, Kanaya AM, Holvoet P, Connelly S, Strotmeyer ES, Harris TB, et al. Association between Oxidized Ldl, Obesity and Type 2 Diabetes in a Population-Based Cohort, the Health, Aging and Body Composition Study. Diabetes Metab Res Rev. 2009;25(8):733-739.

35. Bentley C, Hathaway N, Widdows J, Bejta F, De Pascale C, Avella M, et al. Influence of Chylomicron Remnants on Human Monocyte Activation in Vitro. Nutr Metab Cardiovasc Dis. 2011;21(11):871-878.

36. Astudillo AM, Balgoma D, Balboa MA, Balsinde J. Dynamics of Arachidonic Acid Mobilization by Inflammatory Cells. Biochim Biophys Acta. 2012;1821(2):249-256.

37. Leonard B, Maes M. Mechanistic Explanations How Cell-Mediated Immune Activation, Inflammation and Oxidative and Nitrosative Stress Pathways and Their Sequels and Concomitants Play a Role in the Pathophysiology of Unipolar Depression. Neurosci Biobehav Rev. 2012;36(2):764-785.

38. Kaplan M, Aviram M, Hayek T. Oxidative Stress and Macrophage Foam Cell Formation During Diabetes Mellitus-Induced Atherogenesis: Role of Insulin Therapy. Pharmacol Ther. 2012;136(2):175-185.

39. Mitjavila MT, Moreno JJ. The Effects of Polyphenols on Oxidative Stress and the Arachidonic Acid Cascade. Implications for the Prevention/Treatment of High Prevalence Diseases. Biochem Pharmacol. 2012;84(9):1113-1122.

40. Elmarakby AA, Sullivan JC. Relationship between Oxidative Stress and Inflammatory Cytokines in Diabetic Nephropathy. Cardiovasc Ther. 2012;30(1):49-59.

41. Yudkin JS, Kumari M, Humphries SE, Mohamed-Ali V. Inflammation, Obesity, Stress and Coronary Heart Disease: Is Interleukin-6 the Link? Atherosclerosis. 2000;148(2):209-214.

42. Libby P. What Have We Learned About the Biology of Atherosclerosis? The Role of Inflammation. Am J Cardiol. 2001;88(7B):3J-6J.

43. Schattner M. Role of Nf-Kappab Pathway on Platelet Activation. Circ Res. 2013;113(9):e92.

44. Ghosh S, Hayden MS. New Regulators of Nf-Kappab in Inflammation. Nat Rev Immunol. 2008;8(11):837-848.

45. Lee S, Shin S, Kim H, Han S, Kim K, Kwon J, et al. Anti-Inflammatory Function of Arctiin by Inhibiting Cox-2 Expression Via Nf-Kappab Pathways. J Inflamm (Lond). 2011;8(1):16.

46. Schleicher E, Friess U. Oxidative Stress, Age, and Atherosclerosis. Kidney Int Suppl. 2007(106):S17-26.

47. Uruno A, Yagishita Y, Yamamoto M. The Keap1-Nrf2 System and Diabetes Mellitus. Arch Biochem Biophys. 2015;566:76-84.

48. Cheng X, Siow RC, Mann GE. Impaired Redox Signaling and Antioxidant Gene Expression in Endothelial Cells in Diabetes: A Role for Mitochondria and the Nuclear Factor-E2-Related Factor 2-Kelch-Like Ech-Associated Protein 1 Defense Pathway. Antioxid Redox Signal. 2011;14(3):469-487. 
49. Bhakkiyalakshmi E, Sireesh D, Sakthivadivel M, Sivasubramanian S, Gunasekaran P, Ramkumar KM. Anti-Hyperlipidemic and Anti-Peroxidative Role of Pterostilbene Via Nrf2 Signaling in Experimental Diabetes. Eur J Pharmacol. 2016;777:9-16.

50. Chartoumpekis DV, Kensler TW. New Player on an Old Field; the Keap1/Nrf2 Pathway as a Target for Treatment of Type 2 Diabetes and Metabolic Syndrome. Curr Diabetes Rev. 2013;9(2):137-145.

51. van Buul JD, van Rijssel J, van Alphen FP, van Stalborch AM, Mul EP, Hordijk PL. Icam-1 Clustering on Endothelial Cells Recruits Vcam-1. J Biomed Biotechnol. 2010;2010:120328.

52. Avogaro A, Albiero M, Menegazzo L, de Kreutzenberg S, Fadini GP. Endothelial Dysfunction in Diabetes: The Role of Reparatory Mechanisms. Diabetes Care. 2011;34 Suppl 2:S285-290.

53. Sumagin R, Kuebel JM, Sarelius IH. Leukocyte Rolling and Adhesion Both Contribute to Regulation of Microvascular Permeability to Albumin Via Ligation of Icam-1. Am J Physiol Cell Physiol. 2011;301(4):C804-813.

54. Agarwal B, Campen MJ, Channell MM, Wherry SJ, Varamini B, Davis JG, et al. Resveratrol for Primary Prevention of Atherosclerosis: Clinical Trial Evidence for Improved Gene Expression in Vascular Endothelium. Int J Cardiol. 2013;166(1):246-248.

55. Mochly-Rosen D, Das K, Grimes KV. Protein Kinase C, an Elusive Therapeutic Target? Nat Rev Drug Discov. 2012;11(12):937-957.

56. Koya D, King GL. Protein Kinase C Activation and the Development of Diabetic Complications. Diabetes. 1998;47(6):859-866.

57. Mercado CP, Quintero MV, Li Y, Singh P, Byrd AK, Talabnin K, et al. A Serotonin-Induced NGlycan Switch Regulates Platelet Aggregation. Sci Rep. 2013;3:2795.

58. Forstermann U, Sessa WC. Nitric Oxide Synthases: Regulation and Function. Eur Heart J. 2012;33(7):829-837, 837a-837d.

59. Feletou M, Huang Y, Vanhoutte PM. Endothelium-Mediated Control of Vascular Tone: Cox-1 and Cox-2 Products. Br J Pharmacol. 2011;164(3):894-912.

60. Pulcinelli FM, Biasucci LM, Riondino S, Giubilato S, Leo A, Di Renzo L, et al. Cox-1 Sensitivity and Thromboxane A2 Production in Type 1 and Type 2 Diabetic Patients under Chronic Aspirin Treatment. Eur Heart J. 2009;30(10):1279-1286.

61. Goldberg H, Whiteside C, Fantus IG. O-Linked Beta-N-Acetylglucosamine Supports P38 Mapk Activation by High Glucose in Glomerular Mesangial Cells. Am J Physiol Endocrinol Metab. 2011;301(4):E713-726.

62. Kizub IV, Klymenko KI, Soloviev Al. Protein Kinase C in Enhanced Vascular Tone in Diabetes Mellitus. Int J Cardiol. 2014;174(2):230-242.

63. Boyle AJ, Kelly DJ, Zhang Y, Cox AJ, Gow RM, Way K, et al. Inhibition of Protein Kinase C Reduces Left Ventricular Fibrosis and Dysfunction Following Myocardial Infarction. J Mol Cell Cardiol. 2005;39(2):213-221.

64. Hambleton M, Hahn H, Pleger ST, Kuhn MC, Klevitsky R, Carr AN, et al. Pharmacological- and Gene Therapy-Based Inhibition of Protein Kinase Calpha/Beta Enhances Cardiac Contractility and Attenuates Heart Failure. Circulation. 2006;114(6):574-582.

65. Vandvik PO, Lincoff AM, Gore JM, Gutterman DD, Sonnenberg FA, Alonso-Coello P, et al. Primary and Secondary Prevention of Cardiovascular Disease: Antithrombotic Therapy and Prevention of Thrombosis, 9th Ed: American College of Chest Physicians Evidence-Based Clinical Practice Guidelines. Chest. 2012;141(2 Suppl):e637S-668S.

66. Casellini CM, Barlow PM, Rice AL, Casey M, Simmons K, Pittenger G, et al. A 6-Month, Randomized, Double-Masked, Placebo-Controlled Study Evaluating the Effects of the Protein Kinase C-Beta Inhibitor Ruboxistaurin on Skin Microvascular Blood Flow and Other Measures of Diabetic Peripheral Neuropathy. Diabetes Care. 2007;30(4):896-902.

67. Talior I, Tennenbaum T, Kuroki T, Eldar-Finkelman H. Pkc-Delta-Dependent Activation of Oxidative Stress in Adipocytes of Obese and Insulin-Resistant Mice: Role for Nadph Oxidase. Am J Physiol Endocrinol Metab. 2005;288(2):E405-411. 
68. Cassuto J, Dou HJ, Czikora I, Szabo A, Patel VS, Kamath V, et al. Peroxynitrite Disrupts Endothelial Caveolae Leading to Enos Uncoupling and Diminished Flow-Mediated Dilation in Coronary Arterioles of Diabetic Patients. Diabetes. 2014;63(4):1381-1393.

69. Yang YM, Huang A, Kaley G, Sun D. Enos Uncoupling and Endothelial Dysfunction in Aged Vessels. Am J Physiol Heart Circ Physiol. 2009;297(5):H1829-1836.

70. Forstermann U. Nitric Oxide and Oxidative Stress in Vascular Disease. Pflugers Arch. 2010;459(6):923-939.

71. Tsikas D, Flentje M, Niemann J, Bohmer A, Stichtenoth DO. Extra-Platelet No and No(+)Containing Drugs Are Potent Inhibitors of Platelet Aggregation in Humans by CgmpDependent and Cgmp-Independent Mechanisms. Blood. 2012;119(22):5337-5339; author reply 5339.

72. Napoli C, Ignarro LJ. Nitric Oxide and Pathogenic Mechanisms Involved in the Development of Vascular Diseases. Arch Pharm Res. 2009;32(8):1103-1108.

73. Pirillo A, Norata GD, Catapano AL. Lox-1, OxIdl, and Atherosclerosis. Mediators Inflamm. 2013;2013:152786.

74. Sacerdoti D, Pesce P, Di Pascoli M, Brocco S, Cecchetto L, Bolognesi M. Arachidonic Acid Metabolites and Endothelial Dysfunction of Portal Hypertension. Prostaglandins Other Lipid Mediat. 2015;120:80-90. 
Figure 1. Oxidative stress changing thrombotic state, vascular homeostasis, inflammation and cellular proliferation in T2DM.

Figure 2. Cellular pathways involved in diabetes derived oxidative stress. P65 subunit of NF- $\kappa$ B Pathway increases the gene expression of MCP-1 and VCAM-1 allowing for increased TNF- $\alpha$, white cell adhesion and greater white cell migration leading to vascular inflammation. P65 subunit of NF- $\kappa \mathrm{B}$ also increases cytokine production further upregulating NF- $\mathrm{B}$ and CR-P. Increased ROS synergistically amplifies endothelial dysfunction, AA mobilisation, ONNO- and oxLDL. Increased endothelial dysfunction promotes the production of cellular and platelet adhesion molecules, increasing platelet aggregation and white cell adhesion. Mobilised AA adds to the increase in thromboxane A2 and prostacyclin, altering both platelet aggregation and vessel dilation. ROS activates PK-C Production leading to an increase in cyclooxygenase activity, ET-1, MAP-Kinase and decreasing eNOS causing platelet aggregation, vaso-constriction and vascular inflammation. Methylglyoxal and ROS add to the accumulation in AGE's, creating an increase in AGE-RAGE binding. Binding contributes to vascular proliferation through TGF $\alpha$ and TGF $\beta$ while O-GlcNAcylation and deregulation of enzymatic activity leads to platelet aggregation and the reduction of vascular homeostasis.

AA, Arachidonic acid; AGE, Advanced glycation end-products; C-RP, C-Reactive protein; COX-1, Cyclooxygenase-1; COX-2, Cyclooxygenase-2; ET-1, Endothelin-1; eNOS, Endothelial nitric oxide synthase; ICAM-1, Intercellular adhesion molecule-1; Keap1-NRF2, Kelch-like ECH-associated protein 1-Nuclear factor (erythroid-derived 2)-like 2; MAP-K, Mitogen-activated protein kinase's; MCP-1, Monocyte Chemo-attractant protein-1; NF-кB, Nuclear factor kappa-light-chain-enhancer of activated B cells; NO, Nitric oxide; ONOO-, Peroxynitrite; oxLDL, Oxidised low density lipoproteins; PAI-1, Plasminogen activator inhibitor-1; RAGE, Receptor of Advanced glycation end-products; ROS, Reactive oxygen species; TGF, Transforming growth factor; TNF $\alpha$, Tumour necrosis factor alpha; VCAM-1, Vascular cell adhesion molecule-1; 5-HTEPE, 5Hydroperoxyeicosatetraenoic acid.

Figure 3. Summary of biomarkers implicated by ROS in Platelet hyperactivity, vascular homeostasis, vascular inflammation and cellular proliferation.

AA, Arachidonic acid; AGE, Advanced glycation end-products; C-RP, C-Reactive protein; COX-1, Cyclooxygenase-1; COX-2, Cyclooxygenase-2; EGF, Endothelial growth factor; ET-1, Endothelin-1; eNOS, Endothelial nitric oxide synthase; ICAM-1, Intercellular adhesion molecule-1; IL, Interleukin; INF-I, InterferonI; Keap1-NRF2, Kelch-like ECH-associated protein 1-Nuclear factor (erythroid-derived 2)-like 2; MAF-1, Monocyte activating factor-1; MAP-K, Mitogen-activated protein kinase's; MCP-1, Monocyte Chemo-attractant protein-1; NF-кB, Nuclear factor kappa-light-chain-enhancer of activated B cells; NO, Nitric oxide; ONOO-, Peroxynitrite; oxLDL, Oxidised low density lipoproteins; PAI-1, Plasminogen activator inhibitor-1; PDGF, Platelet-derived growth factor; $\mathrm{PGH} \mathrm{G}_{1}$, Prostaglandin $\mathrm{G}_{1}$; $\mathrm{PGH} \mathrm{H}_{1}$, Prostaglandin $\mathrm{H}_{1}$; PK-C, Protein Kinase-C; RAGE, Receptor of Advanced glycation end-products; ROS, Reactive oxygen species; TGF, Transforming growth factor; TNF $\alpha$, Tumour necrosis factor alpha; VCAM-1, Vascular cell adhesion molecule-1; 5-HTEPE, 5-Hydroperoxyeicosatetraenoic acid.

Figure 1: Simple overview of T2DM induced oxidative stress 


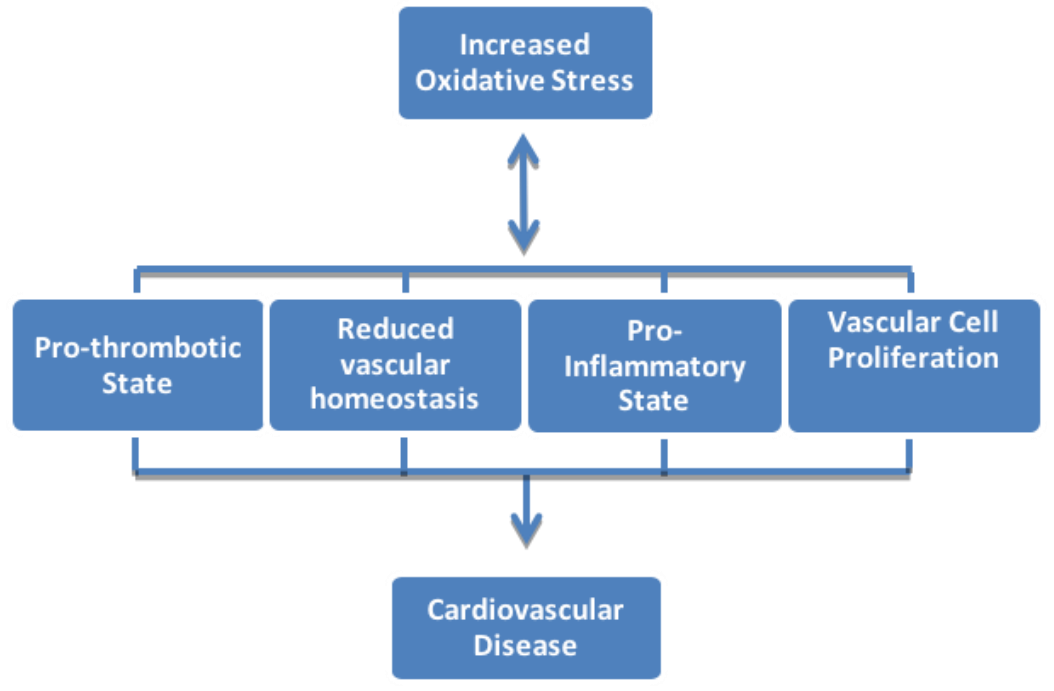

Figure 2. Cellular pathways of ROS induced CVD

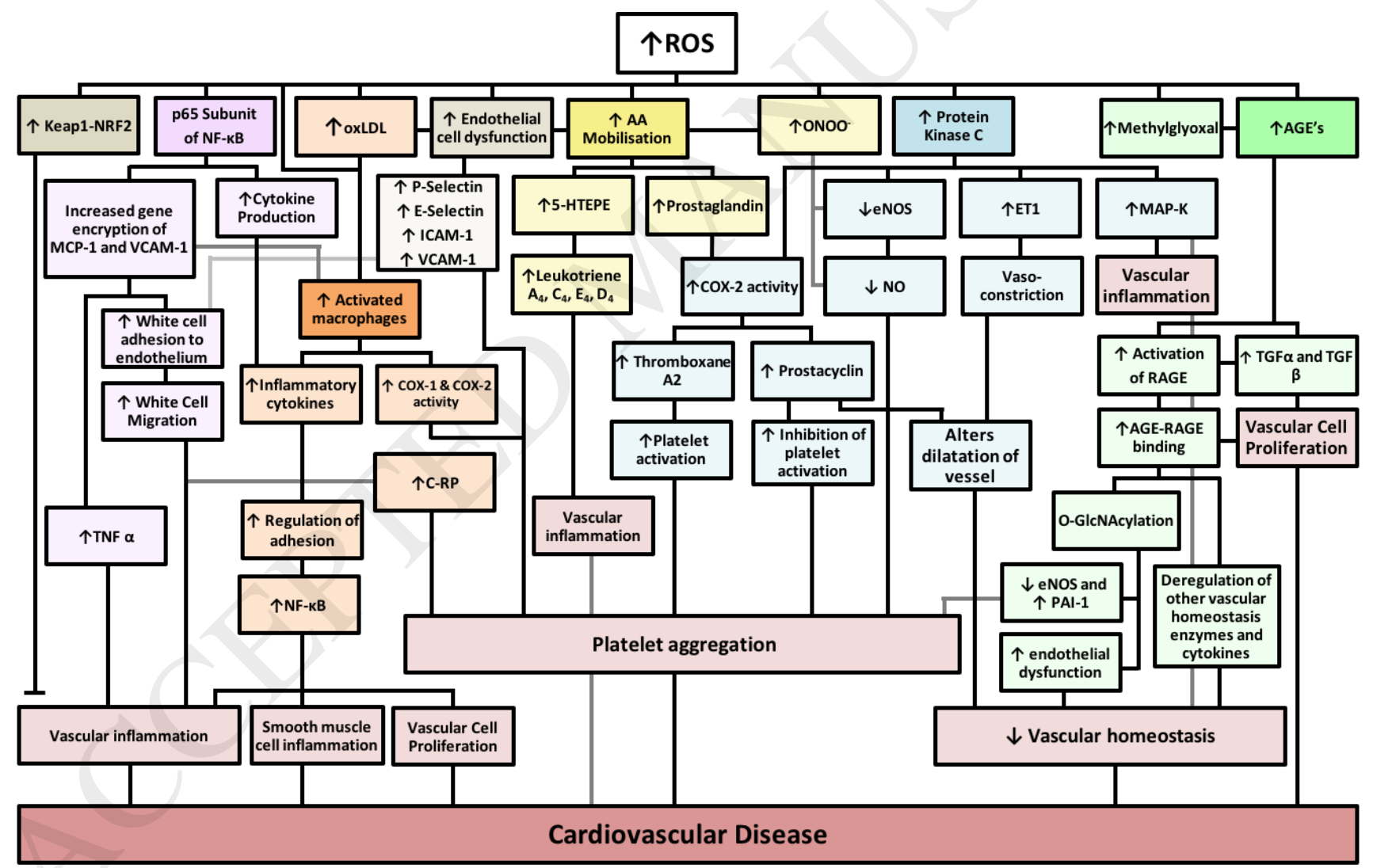


Figure 3. Various biomarkers involved in ROS induced CVD

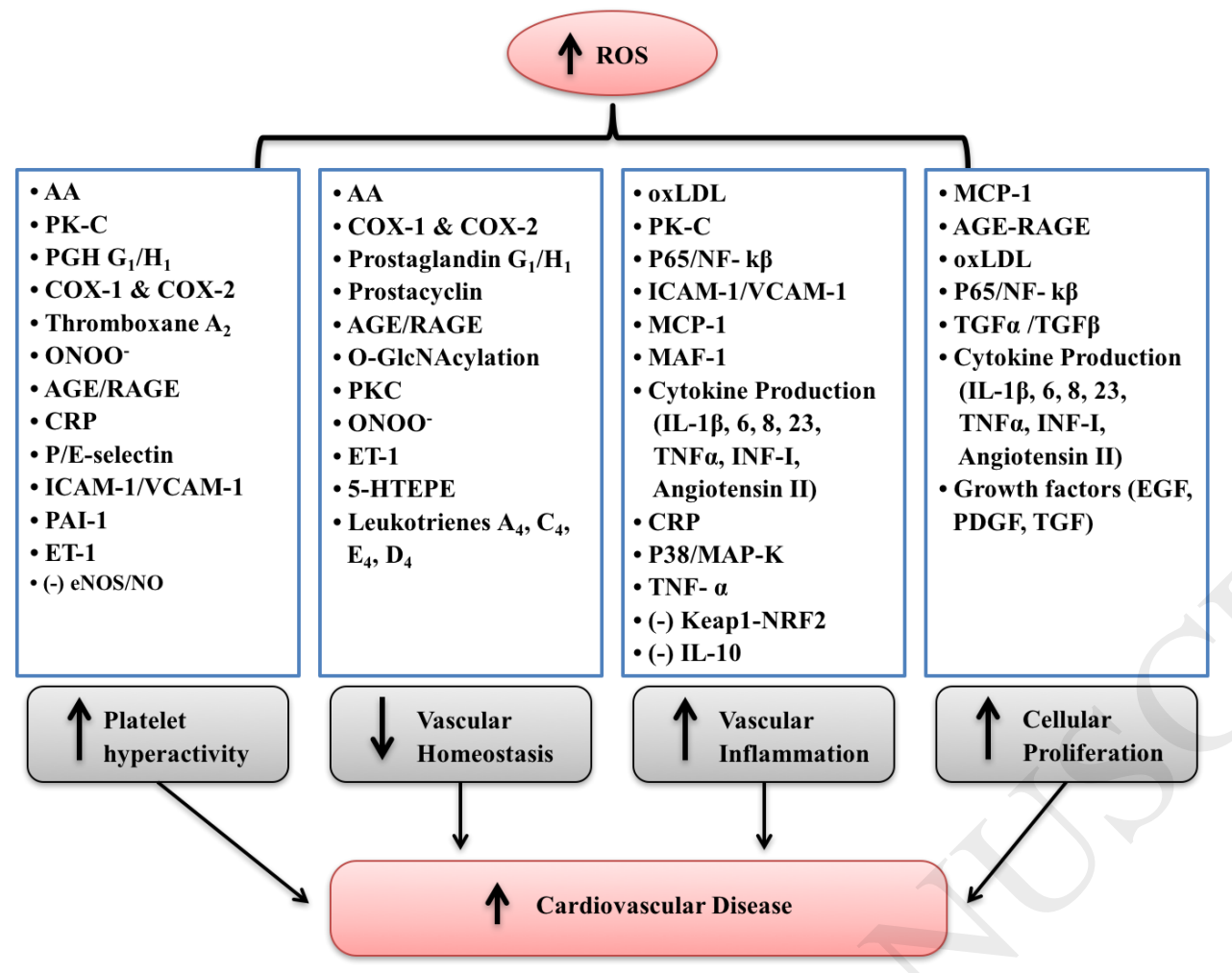




\section{Accepted Manuscript}

Title: OXIDATIVE STRESS BIOMARKERS IN TYPE 2

DIABETES MELLITUS FOR ASSESSMENT OF

CARDIOVASCULAR DISEASE RISK

Authors: Roy Robson, Avinash R. Kundur, Indu Singh

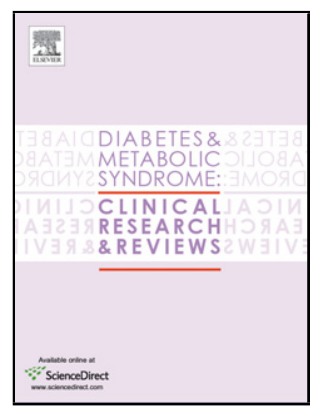

PII:

S1871-4021(17)30465-4

DOI:

https://doi.org/10.1016/j.dsx.2017.12.029

Reference:

DSX 912

To appear in:

Diabetes \& Metabolic Syndrome: Clinical Research \& Reviews

Received date:

$18-12-2017$

Accepted date:

27-12-2017

Please cite this article as: Robson Roy, Kundur Avinash R, Singh Indu.OXIDATIVE STRESS BIOMARKERS IN TYPE 2 DIABETES MELLITUS FOR ASSESSMENT OF CARDIOVASCULAR DISEASE RISK.Diabetes and Metabolic Syndrome: Clinical Research and Reviews https://doi.org/10.1016/j.dsx.2017.12.029

This is a PDF file of an unedited manuscript that has been accepted for publication. As a service to our customers we are providing this early version of the manuscript. The manuscript will undergo copyediting, typesetting, and review of the resulting proof before it is published in its final form. Please note that during the production process errors may be discovered which could affect the content, and all legal disclaimers that apply to the journal pertain. 